\title{
Characterizing the Interplay between Polymer Solvation and Conformation
}

\author{
Debdas Dhabal, Zhitong Jiang, and Amish J. Patel* \\ Department of Chemical and Biomolecular Engineering, University of Pennsylvania, \\ Philadelphia, Pennsylvania 19104, United States
}

E-mail: amish.patel@seas.upenn.edu 


\begin{abstract}
Conformational transitions of flexible molecules, especially those driven by hydrophobic effects, tend to be hindered by desolvation barriers. For such transitions, it is thus important to characterize and understand the interplay between solvation and conformation. Using specialized molecular simulations, here we perform such a characterization for a hydrophobic polymer solvated in water. We find that an external potential, which unfavorably perturbs the polymer hydration waters, can trigger a coilto-globule or collapse transition, and that the relative stabilities of the collapsed and extended states can be quantified by the strength of the requisite potential. Our results also provide mechanistic insights into the collapse transition, highlighting that polymer collapse proceeds through the formation of a sufficiently large non-polar cluster, and that collective water density fluctuations play an important role in stabilizing such a cluster. We also study the collapse of the hydrophobic polymer in octane, a non-polar solvent, and interestingly, we find that the mechanistic details of the transition are qualitatively similar to that in water.
\end{abstract}




\section{Introduction}

Aqueous or organic solutions of conformationally flexible solutes, such as polymers or peptides, play an important role in diverse materials ${ }^{1+4}$ and biomolecular contexts. ${ }^{5}+8$ Although solvated flexible molecules can exist in a multitude of conformations, they often display two or more distinct basins that are separated by free energy barriers, e.g., polymers can be in extended or collapsed states, whereas proteins can be in their native folded structures, become denatured, or even adopt well-defined misfolded configurations. $\stackrel{910}{ }$ Molecular simulations have been used extensively, often with the aid of enhanced sampling methods, to study a variety of flexible molecules, and characterize their conformational free energy landscapes, typically as a function of one or more solute co-ordinates, such as radius of gyration or dihedral angles. $\frac{7111}{71}$

In characterizing such landscapes, solvent degrees of freedom are usually integrated out. Such integration does not lead to any loss of mechanistic information into conformational transitions when solvent degrees of freedom equilibrate rapidly with respect to the conformational co-ordinates. $\frac{12}{2}$ However, certain transitions, such as those driven by hydrophobic effects, tend to feature slow solvent degrees of freedom, which relax on timescales that are comparable to, or even longer than, the relaxation times of the configurational coordinates. $\frac{13}{18}$ In particular, both theoretical and simulation studies have highlighted the importance of slow solvent degrees of freedom in the coil-to-globule transition of non-polar polymers in water. $13|14| 19] 21$

For transitions characterized by slow solvent degrees of freedom, attempts to integrate out the slow solvent co-ordinates can lead to hysteresis in the sampling of the conformational co-ordinates, and make it challenging to accurately estimate the conformational free energy

landscape. ${ }^{22}$ Importantly, the resulting loss of mechanistic information can also obfuscate how the solute conformational landscape might respond to changes in the solvent, e.g., due to the introduction of co-solutes ${ }^{23} \sqrt{26}$ or proximity to interfaces. $27-30$ Thus, characterizing the interplay between conformation and solvation, e.g., through a free energy landscape that 
is a function of both conformational and solvent co-ordinates, can be valuable, particularly

for systems that are expected to feature slow solvent degrees of freedom. ${ }^{1311431}$ However, the enhanced sampling of solvent co-ordinates, which must be performed to obtain such a landscape, can be challenging because the solvation shell of a flexible solute is inherently dynamic, and changes along with the conformation of the solute.

To address this challenge, we recently introduced a method for sampling the number of solvent molecules, $\tilde{N}_{v}$, in a dynamical volume, $v$, which evolves with, and continuously conforms to, the shape of a flexible solute of interest. ${ }^{31}$ Here, we use this method to study the coil-to-globule or collapse transition of a flexible hydrophobic polymer solvated in water. We find that polymer collapse can be triggered by an unfavorable potential, $\phi \tilde{N}_{v}$, that perturbs the polymer hydration waters, $\stackrel{32133}{2}$ and that the potential strength, $\phi$, needed to trigger the transition can serve as a measure of the relative stabilities of the collapsed and extended states. We also characterize the free energy landscape as a function of both the radius of gyration of the polymer (conformational co-ordinate), and the number of waters, $\tilde{N}_{v}$, in its hydration shell (solvent co-ordinate), and use this characterization to uncover mechanistic insights into the collapse transition. We find that polymer collapse proceeds through the formation of a sufficiently large non-polar cluster, and that collective water density fluctuations play an important role in nucleating such a cluster. ${ }^{13114}$ We also study the collapse of the hydrophobic polymer in the non-polar solvent, octane, and find that it is remarkably similar to the collapse of the polymer in water.

\section{Methods}

To interrogate the interplay between the solvation and conformation of flexible solutes, here we study a linear alkane chain consisting of 45 beads that is designated as $\mathrm{C}_{45}$. The solvation shell, $v$, of the $\mathrm{C}_{45}$ chain, is defined as the union of 45 spherical sub-volumes with radius, $r_{v}$, which are pegged to the $\mathrm{C}_{45}$ monomers. Thus, the solvation shell, $v$, of the flexible $\mathrm{C}_{45}$ 
molecule is not static, but changes its shape and/or size dynamically in response to the conformational fluctuations of the polymer. Using the recently developed dynamic INDUS method, $\stackrel{31}{n}$ here we bias the coarse-grained number, $\tilde{N}_{v}$, of solvent heavy atoms in $v ; \tilde{N}_{v}$ is closely related to the actual number of solvent atoms in $v$, but is chosen to be a continuous function of particle positions to permit biasing $\tilde{N}_{v}$ without resulting in impulsive forces. $34 \mid 35$ By using harmonic biasing potentials to sample $\tilde{N}_{v}$ over its range of interest, and using the Weighted Histogram Analysis Method (WHAM) to combine the biased probability distributions, $\stackrel{36}{39}$ we are able to characterize the free energetics, $\beta G_{v}(\tilde{N}) \equiv-\ln P_{v}(\tilde{N})$, where $\beta^{-1} \equiv k_{\mathrm{B}} T$ is the thermal energy, and $P_{v}(\tilde{N})$ is the probability of observing $\tilde{N}$ solvent atoms in $v$. The probability, $P_{v}(\tilde{N}) \equiv\left\langle\delta\left(\tilde{N}-\tilde{N}_{v}\right)\right\rangle_{0}$, is the ensemble average of the Dirac delta function obtained using the unbiased Hamiltonian, $\mathcal{H}_{0}$. Once $G_{v}(\tilde{N})$ is estimated, we use standard reweighting techniques ${ }^{\frac{36}{6}}$ to interrogate how biasing potentials, such as $U_{\phi} \equiv \phi \tilde{N}_{v}$, modulate polymer solvation and determine the corresponding averages, $\langle\ldots\rangle_{\phi}$, obtained using the biased Hamiltonians, $\mathcal{H}_{\phi}=\mathcal{H}_{0}+U_{\phi}$. For example, the free energetics in a biased ensemble, $\beta G_{v}^{\phi}(\tilde{N}) \equiv-\ln P_{v}^{\phi}(\tilde{N})$, can be obtained using $P_{v}^{\phi}(\tilde{N})=\left\langle\delta\left(\tilde{N}-\tilde{N}_{v}\right)\right\rangle_{\phi}$. Finally, to characterize the two-dimensional free energetics, $\beta G_{v}\left(\tilde{N}, R_{\mathrm{g}}\right) \equiv-\ln P_{v}\left(\tilde{N}, R_{\mathrm{g}}\right)$, where $P_{v}\left(\tilde{N}, R_{\mathrm{g}}\right)$ is the joint probability of observing $\tilde{N}$ solvent atoms in $v$, and the polymer with a radius of gyration, $R_{\mathrm{g}}$, we combine the joint probability distributions, observed in our biased simulations, using the WHAM weights determined above. $\frac{35 \mid 40}{1.1}$

\subsection{Simulation Details}

All simulations were performed using GROMACS package ${ }^{41}$ (version 4.5.3), suitably modified to perform indirect umbrella sampling (INDUS) using dynamic probe volumes. $\frac{3135}{\text { The }}$ equations of motion were integrated using the leap-frog algorithm with a time step of 2 fs, and periodic boundary conditions were employed in all three dimensions. The system temperature, $T$, was maintained at $298 \mathrm{~K}$ using the canonical velocity rescaling thermostat $\underline{42}$ with a time constant of $0.5 \mathrm{ps}$, and the system pressure, $P$, was maintained at 1 atm using the 
Parrinello-Rahman barostat ${ }^{43}$ with a time constant of 1 ps. The n-alkane chain containing 45 carbons (designated as $\mathrm{C}_{45}$ ) was modeled using the TraPPE-UA (Transferable Potentials for Phase Equilibria - United Atom) forcefield developed by Siepmann et. al. ${ }^{44}$ The TraPPE-UA forcefield models individual $-\mathrm{CH}_{2^{-}}$or $-\mathrm{CH}_{3}$ units as pseudo-atoms, so that our solute is comprised of 45 united-atom beads. The $\mathrm{C}_{45}$ chain was solvated using either water or n-octane, and Lorentz-Berthelot combination rules were used to determine the Lennard Jones parameters for cross-interactions. In all cases, the simulation box was first energy minimized using the steepest descent algorithm, followed by a 2 ns NVT simulation, and then a 4 ns NPT simulation to equilibrate the system. The biased simulations were run for $10 \mathrm{~ns}$, with either the first $2 \mathrm{~ns}$ (for $\mathrm{C}_{45} /$ water) or $1 \mathrm{~ns}$ (for $\mathrm{C}_{45} /$ octane) being discarded for equilibration; the coarse-grained number of solvent atoms, $\tilde{N}_{v}$, in the polymer hydration shell, $v$, and the polymer radius of gyration, $R_{\mathrm{g}}$, were estimated every $0.1 \mathrm{ps}$. Additional details of the $\mathrm{C}_{45}$ /water and $\mathrm{C}_{45}$ /octane simulations are included below.

$\mathbf{C}_{45}$ /Water: Water was modeled using the SPC/E (extended simple point charge) model ${ }^{45}$ with bonds involving hydrogen atoms being constrained using the SETTLE algorithm. $\underline{46}$ The particle Mesh Ewald (PME) method ${ }^{47}$ was used to compute long-range electrostatic interactions; short-range electrostatic and Lennard Jones interactions were truncated at $1 \mathrm{~nm}$. The $\mathrm{C}_{45}$ chain was hydrated using 11,614 water molecules giving rise to a roughly $7 \mathrm{~nm}$ cubic simulation box. Before performing biased simulations, a $10 \mathrm{~ns}$ long unbiased simulation was performed to estimate the average number of solvent atoms in $v$, and ascertain the $\tilde{N}_{v}$-range that must be sampled; the radius of the spherical sub-volumes used to define $v$ was chosen to be $r_{v}=0.6 \mathrm{~nm}$. The biased simulations used harmonic potentials with a spring constant of $0.0243 \mathrm{~kJ} / \mathrm{mol}$.

$\mathbf{C}_{45}$ /Octane: Octane was modeled using the TraPPE-UA forcefield ${ }^{44}$ with Lennard Jones interactions being truncated at $1.4 \mathrm{~nm}$. The $\mathrm{C}_{45}$ chain was solvated using 1,000 octane molecules. The sub-volume radius used to define $v$ was chosen to be $r_{v}=0.7 \mathrm{~nm}$, and a spring constant of $0.033 \mathrm{~kJ} / \mathrm{mol}$ was used for the harmonic biasing potentials. 


\section{Results and Discussion}

\subsection{How Polymer Hydration Waters Respond to Perturbations}

To characterize how the hydration of the flexible $\mathrm{C}_{45}$ chain influences its conformation, we focus on water molecules in its first hydration shell, $v$ (Figure 1a, cyan/transparent). We account for the dynamic nature of the polymer hydration shell, i.e., changes in its size and shape in response to polymer conformational fluctuations, by defining $v$ to be the union of 45 spherical sub-volumes, where every sub-volume is pegged to a polymer bead, and has a radius, $r_{v}=0.6 \mathrm{~nm}$. We then interrogate how the polymer hydration waters respond to perturbations by employing a biasing potential, $U_{\phi}=\phi \tilde{N}_{v}$, where $\tilde{N}_{v}$ is the (coarse-grained) number of waters in $v$, and $\phi$ is the potential strength. Such a biasing potential provides a convenient way to modulate $\tilde{N}_{v}$, and thereby polymer hydration. In particular, hydration is disfavored for $\phi>0$ with the perturbation becoming more pronounced as $\phi$ is increased; conversely, for $\phi<0$, polymer hydration is favored. From a physical standpoint, a biasing potential of strength, $\phi$, effectively decreases the pressure in the polymer hydration shell by roughly $\phi \rho_{\mathrm{w}}$, where $\rho_{\mathrm{w}}$ is the density of bulk water. $\underline{48}$

The response of the $\mathrm{C}_{45}$ hydration waters to the biasing potential is shown in Figure 1 As expected, the average number of hydration waters, $\left\langle\tilde{N}_{v}\right\rangle_{\phi}$, decreases as $\phi$ is increased (Figure 10). However, the decrease in $\left\langle\tilde{N}_{v}\right\rangle_{\phi}$ is not gradual, but is punctuated by two sharp drops. The corresponding susceptibility, $\partial\left\langle\tilde{N}_{v}\right\rangle_{\phi} / \partial(-\beta \phi)=\left\langle\delta \tilde{N}_{v}^{2}\right\rangle_{\phi}$, shown in Figure 11, displays marked peaks at $\phi$-values, which are denoted by $\phi_{1}^{*}$ and $\phi_{2}^{*}$. The sharp drops in $\left\langle\tilde{N}_{v}\right\rangle_{\phi}$, and the corresponding peaks in susceptibility, suggest that the polymer undergoes collective transitions at potential strengths, $\phi_{1}^{*}$ and $\phi_{2}^{*}$. To shed light on the nature of these transitions, we include representative snapshots of the system for select $\tilde{N}_{v}$-values in Figure 11. Snapshot (I) suggests that the polymer is in an extended configuration for $\phi<\phi_{1}^{*}$; snapshot (II) shows the polymer in a collapsed, but hydrated configuration for $\phi_{1}^{*}<\phi<\phi_{2}^{*}$; and snapshot (III) highlights that the collapsed polymer is dewetted for $\phi>\phi_{2}^{*}$. 

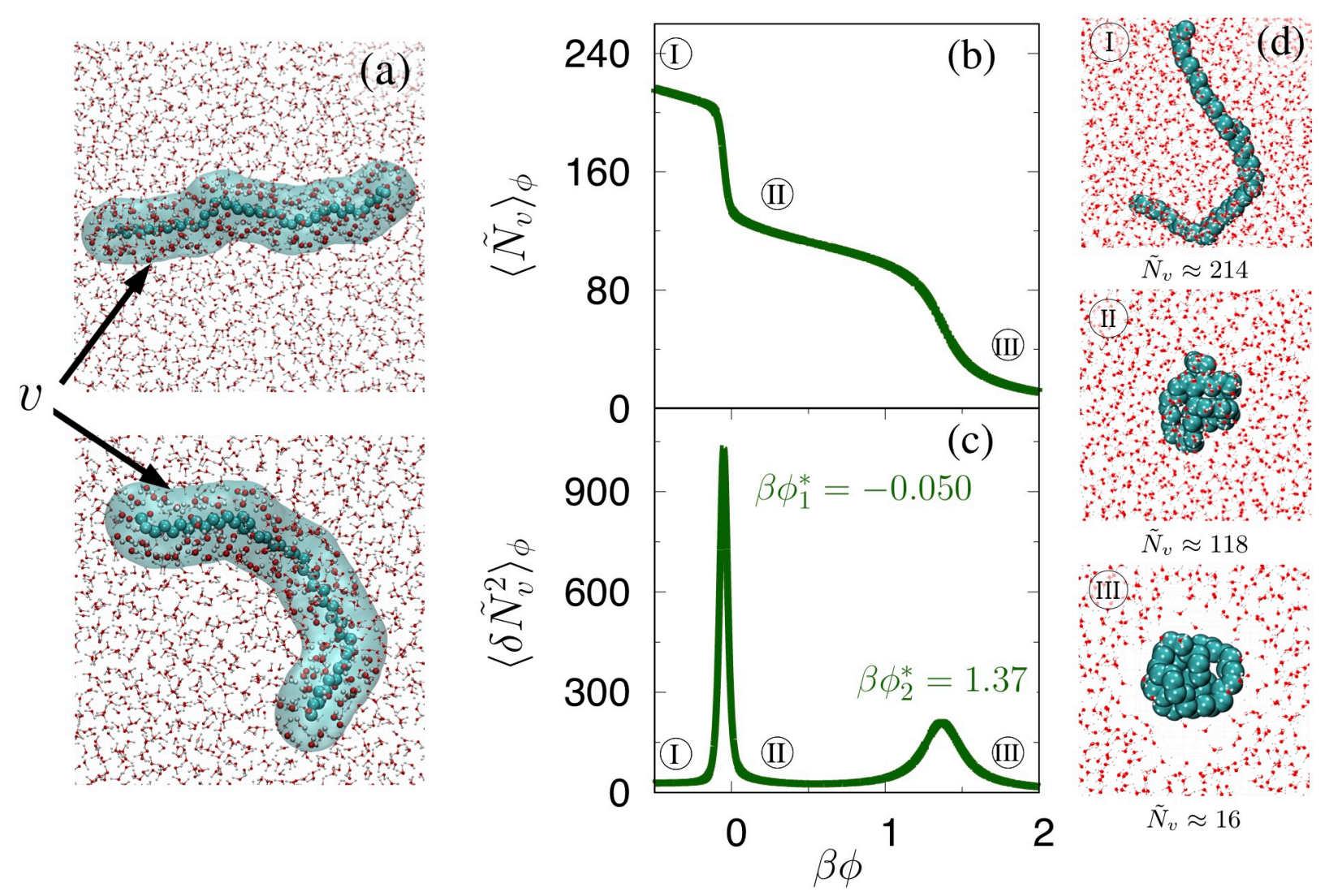

Figure 1: (a) Simulation snapshots of the $C_{45}$ polymer (cyan, space-fill) solvated in water (red/white) are shown. Water molecules in the polymer hydration shell, $v$ (cyan, transparent), are highlighted. As the polymer undergoes conformational fluctuations, both the shape and size of its hydration shell change; to account for such changes, $v$ is defined to be the union of 45 spherical sub-volumes that are centered on the polymer beads and have radii, $r_{v}=0.6 \mathrm{~nm}$. (b) As the strength, $\phi$, of a linear biasing potential, $U_{\phi}=\phi \tilde{N}_{v}$, is increased, the average number of waters, $\left\langle\tilde{N}_{v}\right\rangle_{\phi}$, in the hydration shell decreases; interestingly, two sharp drops are observed in $\left\langle\tilde{N}_{v}\right\rangle_{\phi}$. (c) Correspondingly, the susceptibility, $-\partial\left\langle\tilde{N}_{v}\right\rangle_{\phi} / \partial(\beta \phi)=\left\langle\delta \tilde{N}_{v}^{2}\right\rangle_{\phi}$, displays two peaks at $\phi$-values denoted by $\phi_{1}^{*}$ and $\phi_{2}^{*}$. (d) Simulation snapshots corresponding to three select $\tilde{N}_{v}$-values are shown. As shown in snapshot (I), the polymer adopts an extended configuration for $\tilde{N}_{v}>\left\langle\tilde{N}_{v}\right\rangle_{\phi_{1}^{*}}$, whereas snapshot (II) highlights that the polymer is in a collapsed configuration for $\left\langle\tilde{N}_{v}\right\rangle_{\phi_{2}^{*}}<\tilde{N}_{v}<\left\langle\tilde{N}_{v}\right\rangle_{\phi_{1}^{*}}$. As illustrated in snapshot (III), the collapsed polymer is dewetted for $\tilde{N}_{v}<\left\langle\tilde{N}_{v}\right\rangle_{\phi_{2}^{*}}$.

These snapshots suggest that at potential strength, $\phi_{1}^{*}$, the polymer undergoes a collapse (or folding) transition, whereas at potential strength, $\phi_{2}^{*}$, the collapsed polymer dewets. 

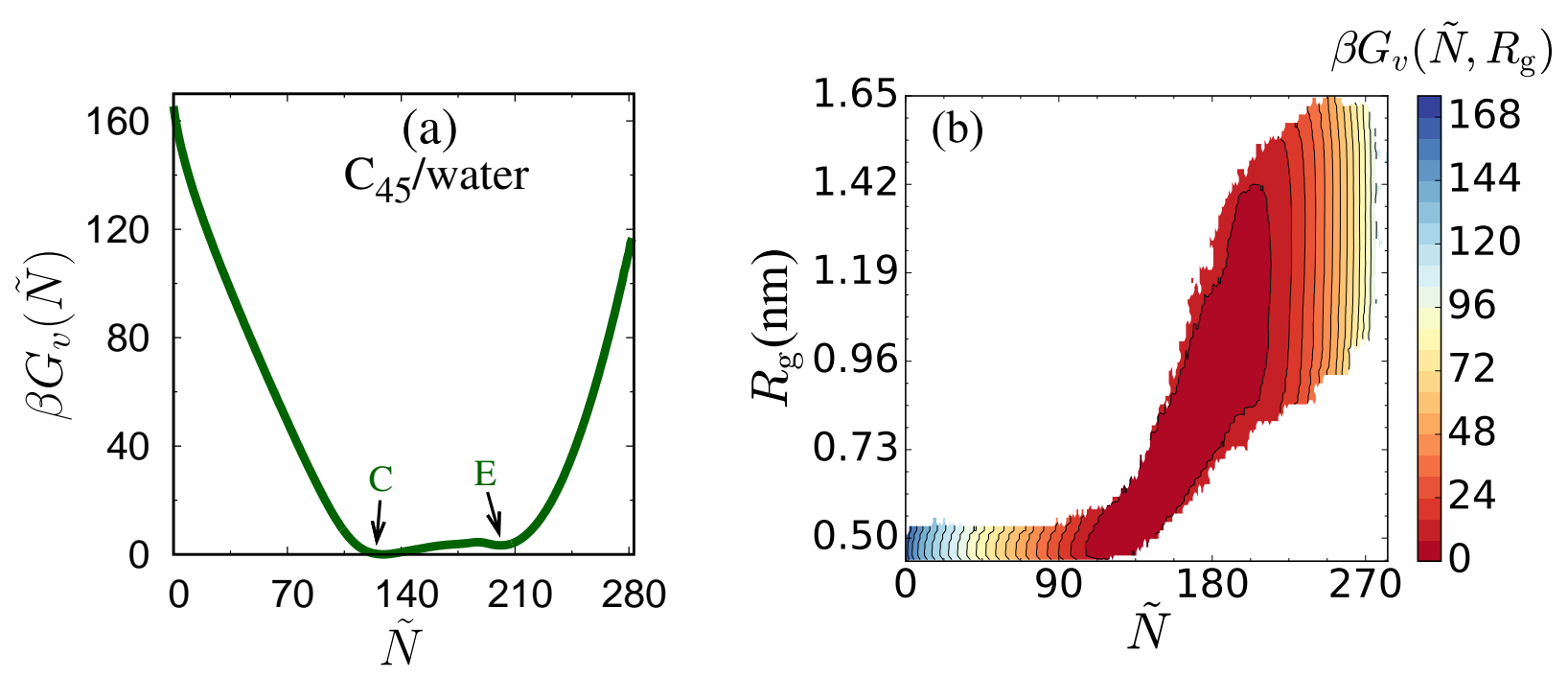

Figure 2: (a) The free energetics of water number fluctuations, $G_{v}(\tilde{N})$, in the dynamical hydration shell of the $C_{45}$ polymer are shown. The basins corresponding to the collapsed (C) and extended (E) states of the polymer are denoted with arrows. (b) To illustrate the interplay between the hydration of the hydrophobic polymer in water and its conformation, we plot the two-dimensional free energy landscape, $G_{v}\left(\tilde{N}, R_{\mathrm{g}}\right)$, where $\tilde{N}$ is the number of waters in $v$, and $R_{\mathrm{g}}$ is the radius of gyration of the $\mathrm{C}_{45}$ polymer. The relatively sharp increase in $G_{v}\left(\tilde{N}, R_{\mathrm{g}}\right)$ at low $\tilde{N}$ and $R_{\mathrm{g}}$ corresponds to the free energetic cost of dewetting the collapsed polymer globule; whereas the increase in $G_{v}\left(\tilde{N}, R_{\mathrm{g}}\right)$ at high $\tilde{N}$ and $R_{\mathrm{g}}$ corresponds to the unfavorable wetting of the extended polymer.

\subsection{Interplay Between Polymer Hydration and Conformation}

To characterize the free energy landscape underpinning the collapse and dewetting transitions, we estimate the free energetics, $G_{v}(\tilde{N})$, of water number fluctuations in the hydration shell of the $\mathrm{C}_{45}$ polymer (Figure $2 \mathrm{a}$ ), as well as the corresponding two-dimensional free energy landscape, $G_{v}\left(\tilde{N}, R_{\mathrm{g}}\right)$ (Figure $\left.2 \mathrm{~b}\right)$. The conformational free energy landscape, $G_{v}\left(R_{\mathrm{g}}\right)$, can be readily obtained from $G_{v}\left(\tilde{N}, R_{\mathrm{g}}\right)$ by integrating out $\tilde{N}$, and is included in Figure $\$ 1$ of the Supplementary Material. Figure 2 confirms the presence of two nearly degenerate basins, observed at low $\tilde{N} \approx 130$ and $R_{\mathrm{g}} \approx 0.5 \mathrm{~nm}$, and at higher $\tilde{N} \approx 200$ and $R_{\mathrm{g}} \approx 1.2 \mathrm{~nm}$, corresponding to the collapsed (C) and extended (E) states, respectively. Figure 2 also suggests that the solvation and conformational co-ordinates are coupled to one another across the collapse transition, and that the transition incurs a free energetic cost of less than $8 k_{\mathrm{B}} T$. In contrast, decreasing $\tilde{N}$ below 100 results in a sharp increase in $G_{v}\left(\tilde{N}, R_{\mathrm{g}}\right)$; such a decrease 
in $\tilde{N}$ is not accompanied by a further decrease in $R_{\mathrm{g}}$, suggesting that it corresponds to the dewetting of the collapsed polymer. Similarly, increasing $\tilde{N}$ above 220 also incurs a large free energetic penalty, and is not accompanied by an increase in $R_{\mathrm{g}}$, suggesting that it represents the unfavorable compression of waters in the hydration shell of the extended polymer.

Although the free energy difference between the collapsed and extended states or the folding free energy, $\Delta G_{\text {fold }}$ is relatively small, Figure $2 \mathrm{a}$ suggests that for the hydrophobic $C_{45}$ chain in water, the collapsed state is nevertheless stable relative to the extended state, with $\beta \Delta G_{\text {fold }}=-3.3$. The relative stability of the collapsed state is also evident from the fact that the potential strength, $\phi_{1}^{*}$, corresponding to the collapse transition is negative (Figure 11c). In fact, as shown below, the two quantities are proportional to one another, with $\Delta G_{\text {fold }} \approx \phi_{1}^{*}\left(\tilde{N}_{\mathrm{E}}-\tilde{N}_{\mathrm{C}}\right)$, where $\tilde{N}_{\mathrm{E}}$ and $\tilde{N}_{\mathrm{C}}$ are $\tilde{N}$-values corresponding to the extended and collapsed basins, respectively. The collapsed and extended states are expected to be

in coexistence with one another in the $\phi_{1}^{*}$-ensemble, i.e., $G_{v}^{\phi_{1}^{*}}\left(\tilde{N}_{\mathrm{C}}\right)=G_{v}^{\phi_{1}^{*}}\left(\tilde{N}_{\mathrm{E}}\right)$, and because $G_{v}^{\phi}(\tilde{N})$ equals $G_{v}(\tilde{N})+\phi \tilde{N}$ within a constant: ${ }^{40} G_{v}\left(\tilde{N}_{\mathrm{C}}\right)+\phi_{1}^{*} \tilde{N}_{\mathrm{C}}=G_{v}\left(\tilde{N}_{\mathrm{E}}\right)+\phi_{1}^{*} \tilde{N}_{\mathrm{E}}$; thus, the polymer folding free energy, $\Delta G_{\text {fold }} \approx G_{v}\left(\tilde{N}_{\mathrm{C}}\right)-G_{v}\left(\tilde{N}_{\mathrm{E}}\right)=\phi_{1}^{*}\left(\tilde{N}_{\mathrm{E}}-\tilde{N}_{\mathrm{C}}\right)$. For the $\mathrm{C}_{45}$ polymer in water, we estimate $\phi_{1}^{*}\left(\tilde{N}_{\mathrm{E}}-\tilde{N}_{\mathrm{C}}\right) \approx-3.47 k_{\mathrm{B}} T$, which in good agreement with $\Delta G_{\text {fold }}$ obtained directly from $G_{v}(\tilde{N})$. Thus, $\phi_{1}^{*}$ can serve as a quantitative measure of $\Delta G_{\text {fold }}$.

\subsection{Free Energy Landscape at the Collapse Transition}

To better understand the transition of the $\mathrm{C}_{45}$ polymer between its extended and collapsed states, we now focus on its behavior in the presence of a biasing potential of strength, $\phi_{1}^{*}$, wherein the two states are expected to be in coexistence with one another. The corresponding free energy landscapes, $G_{v}^{\phi_{1}^{*}}(\tilde{N})$ and $G_{v}^{\phi_{1}^{*}}\left(\tilde{N}, R_{\mathrm{g}}\right)$ are shown in Figure 3. As seen in Figure 3 a, $G_{v}^{\phi_{1}^{*}}(\tilde{N})$ features two basins: the low- $\tilde{N}$, collapsed (C) and the high- $\tilde{N}$, extended (E) basins, which are in coexistence with one another, and are separated by a barrier of roughly $2 k_{\mathrm{B}} T$. Figure 3 also highlights the locations of the basins $(\mathrm{C}, \mathrm{E})$ and the maximum in $G_{v}^{\phi_{1}^{*}}(\tilde{N})$ at intermediate $\tilde{N}$ (I). 

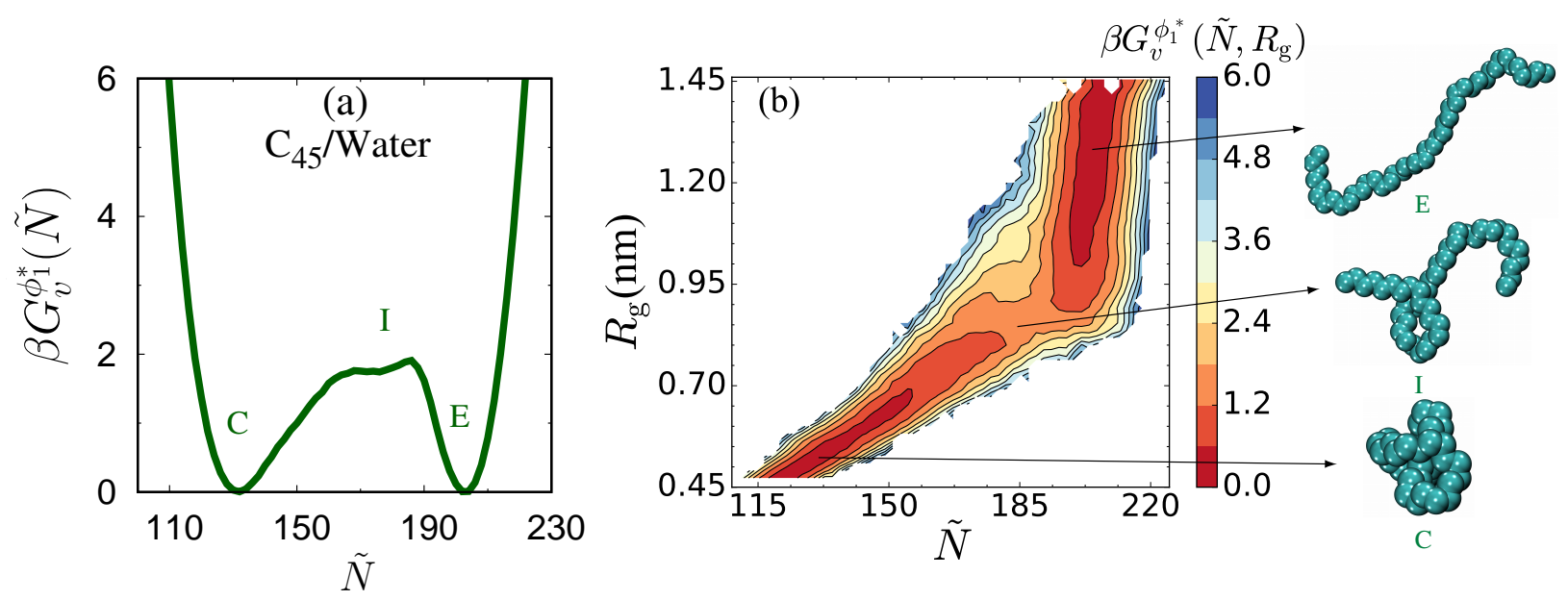

Figure 3: To better understand the coil-to-globule collapse transition of the $\mathrm{C}_{45}$ polymer, here we study its behavior in the $\phi_{1}^{*}$-ensemble; that is, in the presence of the biasing potential, $\phi_{1}^{*} \tilde{N}_{v}$, where $\beta \phi_{1}^{*}=-0.05$. In such a biased ensemble, the coil and globule states are expected to be in coexistence with one another. (a) The free energetics, $G_{v}^{\phi_{1}^{*}}(\tilde{N})$, of water number fluctuations in the $\phi_{1}^{*}$-ensemble, display two distinct basins that are separated by a barrier of roughly $2 k_{\mathrm{B}} T$. The basins corresponding to the collapsed (low $\tilde{N}$ ) and extended (high $\tilde{N}$ ) states of the polymer are labelled " $\mathrm{C}$ " and " $\mathrm{E}$ ", respectively, whereas the location of the maximum in $G_{v}^{\phi_{1}^{*}}(\tilde{N})$ at intermediate $\tilde{N}$ is denoted by "l". (b) The two-dimensional free energy landscape in the $\phi_{1}^{*}$-ensemble, $G_{v}^{\phi_{1}^{*}}\left(\tilde{N}, R_{\mathrm{g}}\right)$, is shown here, and sheds light on the minimum free energy path that the polymer is likely to adopt as it undergoes collapse. Representative configurations of the polymer in the "C", "I", and "E" states are also shown.

The two-dimensional free energy landscape, $G_{v}^{\phi_{1}^{*}}\left(\tilde{N}, R_{\mathrm{g}}\right)$, shown in Figure $3 \mathrm{~b}$, sheds light on the mechanistic pathways that the polymer follows as it undergoes the collapse transition, i.e., it elucidates the minimum free energy path in the $\left(\tilde{N}, R_{\mathrm{g}}\right)$ space, with $\tilde{N}$ and $R_{\mathrm{g}}$ representing the polymer hydration and conformational degrees of freedom. The $G_{v}^{\phi_{1}^{*}}\left(\tilde{N}, R_{\mathrm{g}}\right)$ landscape highlights that in its extended state, the polymer can undergo substantial fluctuations in $R_{\mathrm{g}}$, but only small fluctuations in $\tilde{N}$. To approach the saddle point in $G_{v}^{\phi_{1}^{*}}\left(\tilde{N}, R_{\mathrm{g}}\right)$, the polymer must undergo a marked low- $R_{\mathrm{g}}$ fluctuation, which is followed by a substantive decrease in its hydration waters, $\tilde{N}$. Upon traversing the saddle point, polymer collapse proceeds through a co-ordinated decrease in both $R_{\mathrm{g}}$ and $\tilde{N}$, which is downhill in free energy.

Representative configurations of the polymer in the extended (E), saddle point (I) and collapsed (C) states are also shown Figure $3 \mathrm{~b}$, and shed further light on the pathways involved in polymer collapse. In particular, at the saddle point, the polymer adopts a partially collapsed conformation with some, but not all, of its monomers being clustered together. 
Thus, the formation of a sufficiently large hydrophobic cluster, which not only lowers the $R_{\mathrm{g}}$ of the polymer, but also leads to a decrease in its hydration waters, represents the barrier to polymer collapse. Importantly, as the polymer crosses this barrier, the decrease in its $R_{\mathrm{g}}$ is small relative to the corresponding decrease in its hydration waters, $\tilde{N}$. These findings highlight the importance of collective water density fluctuations in facilitating polymer collapse.

Our results also lend support to the observations of ten Wolde and Chandler, $\frac{13}{13}$ who studied the collapse of a purely repulsive, idealized hydrophobic polymer using a coarse-grained model of water, and those of Miller et al.,, 14 who studied the same polymer using atomistic simulations; both studies found that the formation of a critical non-polar cluster and the associated dewetting represent the barrier to polymer collapse. Moreover, the agreement between these studies, performed on ideal hydrophobic polymers, and our results, obtained using realistic polymers, which have favorable dispersion interactions with water, highlight that weak polymer-water attractions do not qualitatively alter the polymer hydration landscape. $\underline{49}$

\subsection{Polymer Collapse in Octane}

We now study the solvation of the hydrophobic $\mathrm{C}_{45}$ polymer in octane, a non-polar solvent, and draw comparisons to the solvation of the polymer in water. In Figure 4 a, we plot the response of the average number of solvent heavy atoms, $\left\langle\tilde{N}_{v}\right\rangle_{\phi}$, in the polymer solvation shell, $v$, to the strength, $\phi$ of the linear biasing potential, $U_{\phi}=\phi \tilde{N}_{v}$, and in Figure $4 \mathrm{~b}$, we plot the corresponding susceptibility, $-\partial\left\langle\tilde{N}_{v}\right\rangle_{\phi} / \partial(\beta \phi)=\left\langle\delta \tilde{N}_{v}^{2}\right\rangle_{\phi}$. Once again, two sharp drops are observed in $\left\langle\tilde{N}_{v}\right\rangle_{\phi}$ with increasing $\phi$, and correspondingly, two peaks are seen in $\left\langle\delta \tilde{N}_{v}^{2}\right\rangle_{\phi}$ at $\phi$-values denoted by $\phi_{1}^{*}$ and $\phi_{2}^{*}$. The configurations of $\mathrm{C}_{45}$ in octane, shown for three select $\tilde{N}_{v}$-values in Figure $\$ 2$ of the Supplementary Material, suggest that the transitions at $\phi_{1}^{*}$ and $\phi_{2}^{*}$, once again, correspond to polymer collapse and the dewetting of the collapsed polymer. Vertical lines in Figures 4 a,b (blue) correspond to $\phi=0$, and highlight that in contrast with 
$\mathrm{C}_{45}$ in water, $\phi_{1}^{*}$ is positive for $\mathrm{C}_{45}$ in octane. Thus, the folding (or collapse) free energy, $\Delta G_{\text {fold }} \approx \phi_{1}^{*}\left(\tilde{N}_{\mathrm{E}}-\tilde{N}_{\mathrm{C}}\right)$, must also be positive, suggesting that in octane, the extended state of $\mathrm{C}_{45}$ is stable relative to its collapsed state. Figure $4 \mathrm{~b}$ also shows that the dewetting of the collapsed polymer occurs at a lower $\phi_{2}^{*}$-value in octane than in water; this observation can be readily rationalized in terms of the smaller liquid-vapor surface tension of octane, which makes it easier to form cavities. $\frac{50}{5}$
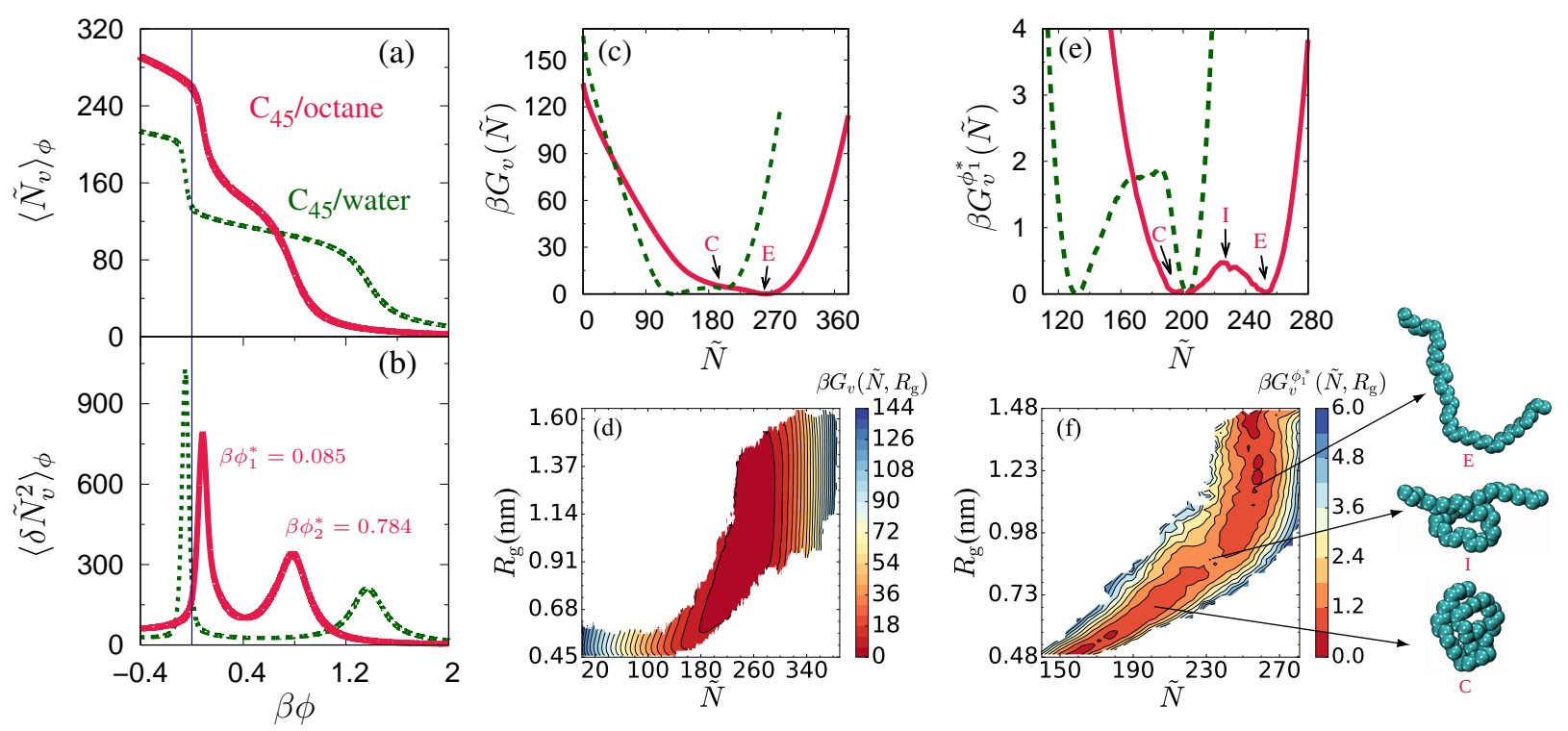

Figure 4: The $\mathrm{C}_{45}$ polymer solvated in octane. (a) The average number of solvent heavy atoms, $\left\langle\tilde{N}_{v}\right\rangle_{\phi}$, in the solvation shell, $v$, of the $\mathrm{C}_{45}$ polymer are shown as a function of the strength, $\phi$, of the linear biasing potential, $U_{\phi}=\phi \tilde{N}_{v}$. For the $C_{45}$ polymer solvated in both octane (red, solid) and in water (green, dashed lines), two sharp drops in $\left\langle\tilde{N}_{v}\right\rangle_{\phi}$ are observed as $\phi$ is increased; the vertical line (blue) depicts $\phi=0$. (b) Correspondingly, the susceptibility, $-\partial\left\langle\tilde{N}_{v}\right\rangle_{\phi} / \partial(\beta \phi)=\left\langle\delta \tilde{N}_{v}^{2}\right\rangle_{\phi}$, displays two peaks in both cases; the locations of the peaks, $\phi_{1}^{*}$ and $\phi_{2}^{*}$, are included for octane. In contrast with $C_{45}$ in water, $\phi_{1}^{*}>0$ for $\mathrm{C}_{45}$ solvated in octane, suggesting that polymer is in its extended state at equilibrium $(\phi=0)$. (c) The free energetics of solvent number fluctuations, $G_{v}(\tilde{N})$, in the hydration shell of the $\mathrm{C}_{45}$ polymer are shown, and the locations of the collapsed (C) and extended (E) states of the polymer are denoted with arrows. In octane, the extended state (high $\tilde{N}$ ) is stable, whereas the collapsed state (low $\tilde{N})$ is unstable. (d) The two-dimensional free energy landscape, $G_{v}\left(\tilde{N}, R_{\mathrm{g}}\right)$, for the $\mathrm{C}_{45}$ polymer solvated in octane is shown. (e) The free energetics, $G_{v}^{\phi_{1}^{*}}(\tilde{N})$, of solvent number fluctuations in the $\phi_{1}^{*}$ ensemble, display two distinct basins that are separated by a barrier. ( $f$ ) The two-dimensional free energy landscape, $G_{v}^{\phi_{1}^{*}}\left(\tilde{N}, R_{\mathrm{g}}\right)$, shown here for the $\mathrm{C}_{45}$ polymer solvated in octane, as well as the representative configurations of the polymer, are qualitatively similar to those for $C_{45}$ in water (Figure $3 \mathrm{~b}$ ).

The unbiased free energy landscapes, $G_{v}(\tilde{N})$ and $G_{v}\left(\tilde{N}, R_{\mathrm{g}}\right)$, for $\mathrm{C}_{45}$ in octane, are shown in Figures 4, d, and the conformational free energy landscape, $G_{v}\left(R_{\mathrm{g}}\right)$, is included as Fig- 
ure $\mathrm{S} 3$ of the Supplementary Material. Apart from the fact that the collapsed state is metastable with respect to the extended state, with $\beta \Delta G_{\text {fold }}=4.5$, the landscapes are qualitatively similar to those for $\mathrm{C}_{45}$ in water (Figure 2). The corresponding free energy landscapes in the $\phi_{1}^{*}$-ensemble are shown in Figures 4 ,f. As with $\mathrm{C}_{45}$ in water, the collapsed (C) and extended (E) states are degenerate in the $\phi_{1}^{*}$-ensemble, and a barrier is observed at intermediate $\tilde{N}$ and $R_{\mathrm{g}}$ for $\mathrm{C}_{45}$ in octane. Although the barrier height is somewhat lower for $\mathrm{C}_{45}$ in octane (relative to water), both the $G_{v}^{\phi_{1}^{*}}\left(\tilde{N}, R_{\mathrm{g}}\right)$ landscape and representative configurations of the polymer (Figure $4 \mathrm{f}$ ), suggest that polymer collapse in octane proceeds through mechanistic pathways, which are remarkably similar to those in water (Figure $3 \mathrm{~b}$ ). In particular, as in water, the collapse of the $\mathrm{C}_{45}$ polymer in octane also proceeds through the formation of a sufficiently large cluster, which is both partially collapsed and partially desolvated.

To understand these similarities, we first recognize that polymer collapse involves the solvent-mediated assembly of well-solvated sub-units, which are smaller than $1 \mathrm{~nm}$, into a cluster that is larger than $1 \mathrm{~nm}$ in size. $\frac{5152}{152}$ According to the Lum-Chandler-Weeks theory of hydrophobicity, 19 the solvation free energy of the former scales as their excluded volume, whereas the solvation free energy of the latter scales as its surface area: $\frac{53+57}{57}$ this interplay between solvation at small and large length scales is responsible for the emergence of a critical cluster, which must be nucleated for the collapse transition to proceed. $\frac{1314}{14}$ Interestingly, a solute size-dependent crossover in solvation free energies has also been observed in non-polar solvents, such as octane. $\frac{50158}{}$ Although the corresponding solvophobic effect, experienced by solutes in non-polar solvents, is expected to be weaker than the hydrophobic effect, $\stackrel{5459}{\text { our }}$ results suggest that it may nevertheless be responsible for the qualitative similarities between the polymer collapse pathways in water and in octane. 


\section{Conclusions and Outlook}

Using the recently developed dynamic Indirect Umbrella Sampling (INDUS) method for sampling the number of solvent molecules, $\tilde{N}_{v}$, in the dynamical solvation shell of a flexible solute, here we study the coil-to-globule or collapse transition of a hydrophobic polymer solvated in water and in the non-polar solvent, octane. We find that an unfavorable potential, which perturbs $\tilde{N}_{v}$, can trigger the collapse transition, and that the requisite potential strength quantifies the relative stabilities of the collapsed and extended states. To understand the interplay between polymer hydration and conformation, we characterize the free energy landscape of the system as a function of both the number of solvent molecules, $\tilde{N}_{v}$, in the polymer hydration shell, and its radius of gyration, $R_{\mathrm{g}}$. We find that polymer collapse proceeds through the formation of a critical non-polar cluster, which represents the bottleneck to the coil-to-globule transition. In particular, assisted by thermal fluctuations, an extended polymer must first adopt a relatively compact configuration, which then undergoes collective dewetting; if the resulting non-polar cluster is sufficiently large, the rest of the polymer then collapses spontaneously.

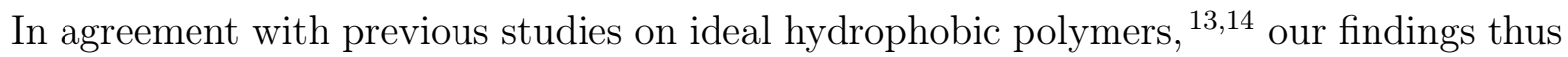
confirm the presence of slow solvent co-ordinates in the coil-to-globule transition, and highlight the importance of collective solvent density fluctuations in overcoming the corresponding barriers. In contrast with water, which is a poor solvent for the hydrophobic polymer, the non-polar solvent, octane, is expected to be a good solvent for the polymer. Interestingly, we find that the mechanistic details of the collapse transition in octane are nevertheless remarkably similar to that in water. Our results suggest that the size-dependent crossover in solvation free energies, which has been observed not just in water, $\sqrt[54]{4}$ but also in non-polar solvents, such as octane, ${ }^{50 \mid 58}$ may underpin the similar polymer collapse pathways in these two solvents.

A characterization of the free energetics of water density fluctuations in static volumes,

both in bulk water $\sqrt[5360]{60}$ and in the vicinity of surfaces,,$\frac{61] 64}{64}$ has provided numerous insights 
into the collective, solvent-mediated hydrophobic effects, ranging from micelle formation and interfacial assembly, to superhydrophobicity and protein interactions. .65696 By characterizing the free energetics of solvent density fluctuations in the dynamic solvation shells of conformationally flexible molecules, we hope that our work will similarly pave the way for a richer understanding of the conformational transitions ${ }^{70171}$ and the phase behavior $\frac{72173}{7}$ of a large classes of interesting solutes, ranging from hydrocarbons and polymers, to peptides

and nucleic acids. $\frac{30|31| 74}{1}$ Our approach is also likely to shed light into how co-solutes (e.g., osmolytes, salts, etc.), which have a propensity to be included or excluded from the vicinity of a polymer, might modulate the solvation of a polymer as well as its conformational landscape. $\frac{75}{80}$

\section{Acknowledgement}

A.J.P. gratefully acknowledges financial support from the National Science Foundation (NSF grants CBET-1652646, CHE-1665339 and DMR-1720530), and awards from the Alfred P. Sloan Research Foundation (FG-2017-9406) and the Camille and Henry Dreyfus Foundation (TC-19-033). D.D. was supported by NSF grant CHE-1665339. The authors thank Akash Pallath for helpful discussions.

\section{References}

(1) Hu, D.; Yu, J.; Wong, K.; Bagchi, B.; Rossky, P. J.; Barbara, P. F. Collapse of stiff conjugated polymers with chemical defects into ordered, cylindrical conformations. Nature 2000, 405, 1030-1033.

(2) Lin, B.; Martin, T. B.; Jayaraman, A. Decreasing Polymer Flexibility Improves Wetting and Dispersion of Polymer-Grafted Particles in a Chemically Identical Polymer Matrix. ACS Macro Letters 2014, 3, 628-632. 
(3) Pandav, G.; Pryamitsyn, V.; Errington, J.; Ganesan, V. Multibody Interactions, Phase Behavior, and Clustering in Nanoparticle-Polyelectrolyte Mixtures. J. Phys. Chem. B. 2015, 119, 14536-14550.

(4) Sharma, A.; Liu, L.; Parameswaran, S.; Grayson, S. M.; Ashbaugh, H. S.; Rick, S. W. Design of Amphiphilic Polymers via Molecular Dynamics Simulations. J. Phys. Chem. B 2016, 120, 10603-10610.

(5) Thirumalai, D.; Lorimer, G. H. Chaperonin-Mediated Protein Folding. Annu. Rev. Biophys. Biomol. Str. 2001, 30, 245-269.

(6) Matysiak, S.; Debenedetti, P. G.; Rossky, P. J. Dissecting the Energetics of Hydrophobic Hydration of Polypeptides. J. Phys. Chem. B. 2011, 115, 14859-14865.

(7) Carmichael, S. P.; Shell, M. S. A New Multiscale Algorithm and Its Application to Coarse-Grained Peptide Models for Self-Assembly. J. Phys. Chem. B. 2012, 116, 83838393.

(8) Bellissent-Funel, M.-C.; Hassanali, A.; Havenith, M.; Henchman, R.; Pohl, P.; Sterpone, F.; van der Spoel, D.; Xu, Y.; Garcia, A. E. Water Determines the Structure and Dynamics of Proteins. Chem. Rev. 2016, 116, 7673-7697.

(9) DuBay, K. H.; Bowman, G. R.; Geissler, P. L. Fluctuations within Folded Proteins: Implications for Thermodynamic and Allosteric Regulation. Acc. Chem. Res. 2015, 48, 1098-1105.

(10) Peter, E. K.; Shea, J.-E.; Pivkin, I. V. Coarse kMC-based replica exchange algorithms for the accelerated simulation of protein folding in explicit solvent. Phys. Chem. Chem. Phys. 2016, 18, 13052-13065.

(11) Lawrence, C. W.; Kumar, S.; Noid, W. G.; Showalter, S. A. Role of Ordered Proteins 
in the Folding-Upon-Binding of Intrinsically Disordered Proteins. J. Phys. Chem. Lett. 2014, 5, 833-838.

(12) Ferguson, A. L.; Panagiotopoulos, A. Z.; Debenedetti, P. G.; Kevrekidis, I. G. Systematic determination of order parameters for chain dynamics using diffusion maps. Proc. Natl. Acad. Sci. U.S.A. 2010, 107, 13597-13602.

(13) ten Wolde, P. R.; Chandler, D. Drying-induced hydrophobic polymer collapse. Proc. Natl. Acad. Sci. U.S.A. 2002, 99, 6539-6543.

(14) Miller, T. F.; Vanden-Eijnden, E.; Chandler, D. Solvent coarse-graining and the string method applied to the hydrophobic collapse of a hydrated chain. Proc. Natl. Acad. Sci. U.S.A. 2007, 104, 14559-14564.

(15) Berne, B. J.; Weeks, J. D.; Zhou, R. Dewetting and Hydrophobic Interaction in Physical and Biological Systems. Annu. Rev. Phys. Chem. 2009, 60, 85-103.

(16) Setny, P.; Baron, R.; Michael Kekenes-Huskey, P.; McCammon, J. A.; Dzubiella, J. Solvent fluctuations in hydrophobic cavity-ligand binding kinetics. Proc. Natl. Acad. Sci. U.S.A. 2013, 110, 1197-1202.

(17) Remsing, R. C.; Xi, E.; Vembanur, S.; Sumit, S.; Debenedetti, P. G.; Garde, S.; Patel, A. J. Pathways to dewetting in hydrophobic confinement. Proc. Natl. Acad. Sci. U.S.A. 2015, 8181-8186, 112.

(18) Tiwary, P.; Mondal, J.; Morrone, J. A.; Berne, B. Role of Water and Steric Constraints in the Kinetics of Cavity-Ligand Unbinding. Proc. Natl. Acad. Sci. U.S.A. 2015, 112, $12015-12019$.

(19) Lum, K.; Chandler, D.; Weeks, J. D. Hydrophobicity at small and large length scales. J. Phys. Chem. B 1999, 103, 4570-4577. 
(20) Athawale, M. V.; Goel, G.; Ghosh, T.; Truekett, T. M.; Garde, S. Effects of lengthscales and attractions on the collapse of hydrophobic polymers in water. Proc. Natl. Acad. Sci. U.S.A. 2007, 104, 733-738.

(21) Goel, G.; Athawale, M. V.; Garde, S.; Truskett, T. M. Attractions, Water Structure, and Thermodynamics of Hydrophobic Polymer Collapse. J. Phys. Chem. B 2008, 112, $13193-13196$.

(22) Xi, E.; Marks, S. M.; Fialoke, S.; Patel, A. J. Sparse sampling of water density fluctuations near liquid-vapor coexistence. Mol. Simul. 2018, 44, 1124-1135.

(23) Athawale, M. V.; Sarupria, S.; Garde, S. Enthalpy-Entropy Contributions to Salt and Osmolyte Effects on Molecular-Scale Hydrophobic Hydration and Interactions. J. Phys. Chem. B 2008, 112, 5661-5670.

(24) Stirnemann, G.; Kang, S.-g.; Zhou, R.; Berne, B. J. How force unfolding differs from chemical denaturation. Proc. Natl. Acad. Sci. U.S.A. 2014, 111, 3413-3418.

(25) Mondal, J.; Halverson, D.; Li, I. T. S.; Stirnemann, G.; Walker, G. C.; Berne, B. How osmolytes influence hydrophobic polymer conformations: A unified view from experiment and theory. Proc. Natl. Acad. Sci. U.S.A. 2015, 112, 9270-9275.

(26) Nayar, D.; van der Vegt, N. F. A. Cosolvent Effects on Polymer Hydration Drive Hydrophobic Collapse. J. Phys. Chem. B. 2018, 122, 3587-3595.

(27) Jamadagni, S. N.; Godawat, R.; Dordick, J. S.; Garde, S. How Interfaces Affect Hydrophobically Driven Polymer Folding. J. Phys. Chem. B 2009, 113, 4093-4101, PMID: 19425248.

(28) Jamadagni, S. N.; Godawat, R.; Garde, S. How Surface Wettability Affects the Binding, Folding, and Dynamics of Hydrophobic Polymers at Interfaces. Langmuir 2009, 25, 13092-13099. 
(29) Vembanur, S.; Patel, A. J.; Sarupria, S.; Garde, S. On the Thermodynamics and Kinetics of Hydrophobic Interactions at Interfaces. J. Phys. Chem. B 2013, 117, 1026110270.

(30) Zerze, G. H.; Mullen, R. G.; Levine, Z. A.; Shea, J.-E.; Mittal, J. To What Extent Does Surface Hydrophobicity Dictate Peptide Folding and Stability near Surfaces? Langmuir 2015, 31, 12223-12230.

(31) Jiang, Z.; Remsing, R. C.; Rego, N. B.; Patel, A. J. Characterizing Solvent Density Fluctuations in Dynamical Observation Volumes. J. Phys. Chem. B 2019, 123, 16501661.

(32) Patel, A. J.; Varilly, P.; Jamadagni, S. N.; Hagan, M. F.; Chandler, D. Sitting at the Edge: How Biomolecules use Hydrophobicity to Tune Their Interactions and Function. J. Phys. Chem. B 2012, 116, 2498-2503.

(33) Patel, A. J.; Garde, S. Efficient Method To Characterize the Context-Dependent Hydrophobicity of Proteins. J. Phys. Chem. B 2014, 118, 1564-1573.

(34) Patel, A. J.; Varilly, P.; Chandler, D. Fluctuations of Water near Extended Hydrophobic and Hydrophilic Surfaces. J. Phys. Chem. B 2010, 114, 1632-1637.

(35) Patel, A. J.; Varilly, P.; Chandler, D. Quantifying Density Fluctuations in Volumes of All Shapes and Sizes Using Indirect Umbrella Sampling. J. Stat. Phys. 2011, 145, $265-275$.

(36) Souaille, M.; Roux, B. Extension to the weighted histogram analysis method: combining umbrella sampling with free energy calculations. Comput. Phys. Commun. 2001, 135, $40-57$.

(37) Shirts, M. R.; Chodera, J. D. Statistically optimal analysis of samples from multiple equilibrium states. J. Chem. Phys. 2008, 129, 124105. 
(38) Zhu, F.; Hummer, G. Convergence and error estimation in free energy calculations using the weighted histogram analysis method. J. Comput. Chem. 2012, 33, 453-465.

(39) Tan, Z.; Gallichio, E.; Lapelosa, M.; Levy, R. M. Theory of binless multi-state free energy estimation with applications to protein-ligand binding. J. Chem. Phys. 2012, $136,144102$.

(40) Xi, E.; Remsing, R. C.; Patel, A. J. Sparse Sampling of Water Density Fluctuations in Interfacial Environments. J. Chem. Theory Comput. 2016, 12, 706-713.

(41) Hess, B.; Kutzner, C.; van der Spoel, D.; Lindahl, E. GROMACS 4: Algorithms for Highly Efficient, Load-Balanced, and Scalable Molecular Simulation. J. Chem. Theory Comput. 2008, 4, 435-447.

(42) Bussi, G.; Donadio, D.; Parrinello, M. Canonical Sampling through Velocity Rescaling. J. Chem. Phys. 2007, 126, 014101.

(43) Parrinello, M.; Rahman, A. Polymorphic Transitions in Single Crystals: A New Molecular Dynamics Method. J. Appl. Phys. 1981, 52, 7182-7190.

(44) Martin, M. G.; Siepmann, J. I. Transferable Potentials for Phase Equilibria. 1. UnitedAtom Description of n-Alkanes. J. Phys. Chem. B. 1998, 102, 2569-2577.

(45) Berendsen, H. J. C.; Grigera, J. R.; Straatsma, T. P. The Missing Term in Effective Pair Potentials. J. Phys. Chem. 1987, 91, 6269-6271.

(46) Miyamoto, S.; Kollman, P. A. Settle: An analytical version of the SHAKE and RATTLE algorithm for rigid water models. J. Comput. Chem. 1992, 13, 952-962.

(47) Essmann, U.; Perera, L.; Berkowitz, M. L.; Darden, T.; Lee, H.; Pedersen, L. G. A Smooth Particle Mesh Ewald Method. J. Chem. Phys. 1995, 103, 8577-8593.

(48) Rego, N. B.; Xi, E.; Patel, A. J. Protein Hydration Waters are Susceptible to Unfavourable Perturbations. J. Am. Chem. Soc. 2019, 141, 2080-2086. 
(49) Remsing, R. C.; Patel, A. J. Water density fluctuations relevant to hydrophobic hydration are unaltered by attractions. J. Chem. Phys. 2015, 142, 024502.

(50) Wu, E.; Garde, S. Lengthscale-Dependent Solvation and Density Fluctuations in nOctane. J. Phys. Chem. B 2015, 119, 9287-9294.

(51) Li, I. T. S.; Walker, G. C. Signature of hydrophobic hydration in a single polymer. Proc. Natl. Acad. Sci. U.S.A. 2011, 108, 16527-16532.

(52) Garde, S.; Patel, A. J. Unraveling the hydrophobic effect, one molecule at a time. Proc. Natl. Acad. Sci. U.S.A. 2011, 108, 16491-16492.

(53) Hummer, G.; Garde, S.; Garcia, A. E.; Pohorille, A.; Pratt, L. R. An Information Theory Model of Hydrophobic Interactions. Proc. Natl. Acad. Sci. U.S.A. 1996, 93, $8951-8955$.

(54) Rajamani, S.; Truskett, T. M.; Garde, S. Hydrophobic hydration from small to large lengthscales: Understanding and manipulating the crossover. Proc. Natl. Acad. Sci. U.S.A. 2005, 102, 9475-9480.

(55) Varilly, P.; Patel, A. J.; Chandler, D. An improved coarse-grained model of solvation and the hydrophobic effect. J. Chem. Phys. 2011, 134, 074109.

(56) Vaikuntanathan, S.; Rotskoff, G.; Hudson, A.; Geissler, P. L. Necessity of Capillary Modes in a Minimal Model of Nanoscale Hydrophobic Solvation. Proc. Natl. Acad. Sci. U.S.A. 2016, 113, E2224-E2230.

(57) Xi, E.; Patel, A. J. The hydrophobic effect, and fluctuations: the long and the short of it. Proc. Natl. Acad. Sci. U.S.A. 2016, 113, 4549-4551.

(58) Huang, D. M.; Chandler, D. Cavity formation and the drying transition in the LennardJones fluid. Phys. Rev. E 2000, 61, 1501-1506. 
(59) Cerdeirina, C. A.; Debenedetti, P. G.; Rossky, P. J.; Giovambattista, N. Evaporation Length Scales of Confined Water and Some Common Organic Liquids. J. Phys. Chem. Lett. 2011, 2, 1000-1003.

(60) Garde, S.; Hummer, G.; Garica, A. E.; Paulaitis, M. E.; Pratt, L. R. Origin of Entropy Convergence in Hydrophobic Hydration and Protein Folding. Phys. Rev. Lett. 1996, rॅ, 4966.

(61) Godawat, R.; Jamadagni, S. N.; Garde, S. Characterizing hydrophobicity of interfaces by using cavity formation, solute binding, and water correlations. Proc. Natl. Acad. Sci. U.S.A. 2009, 106, 15119-15124.

(62) Acharya, H.; Vembanur, S.; Jamadagni, S. N.; Garde, S. Mapping Hydrophobicity at the Nanoscale: Applications to Heterogeneous Surfaces and Proteins. Faraday Discuss. 2010, 146, 353-365.

(63) Rotenberg, B.; Patel, A. J.; Chandler, D. Molecular Explanation for Why Talc Surfaces can be Both Hydrophilic and Hydrophobic. J. Am. Chem. Soc. 2011, 133, 20521 20527 .

(64) Jamadagni, S. N.; Godawat, R.; Garde, S. Hydrophobicity of Proteins and Interfaces: Insights from Density Fluctuations. Annu. Rev. Chem. Biomol. Eng. 2011, 2, 147-171.

(65) Maibaum, L.; Dinner, A. R.; Chandler, D. Micelle Formation and the Hydrophobic Effect. J. Phys. Chem. B 2004, 108, 6778-6781.

(66) Patel, A. J.; Varilly, P.; Jamadagni, S. N.; Acharya, H.; Garde, S.; Chandler, D. Extended surfaces modulate hydrophobic interactions of neighboring solutes. Proc. Natl. Acad. Sci. U.S.A. 2011, 108, 17678-17683.

(67) Prakash, S.; Xi, E.; Patel, A. J. Spontaneous recovery of superhydrophobicity on nanotextured surfaces. Proc. Natl. Acad. Sci. U.S.A. 2016, 113, 5508-5513. 
(68) Xi, E.; Venkateshwaran, V.; Li, L.; Rego, N.; Patel, A. J.; Garde, S. Hydrophobicity of proteins and nanostructured solutes is governed by topographical and chemical context. Proc. Natl. Acad. Sci. U.S.A. 2017, 114, 13345-13350.

(69) Rego, N. B.; Xi, E.; Patel, A. J. Identifying hydrophobic protein patches to inform protein interaction interfaces. Proc. Natl. Acad. Sci. U.S.A. 2021, 118, e2018234118.

(70) Rodriguez-Ropero, F.; Hajari, T.; van der Vegt, N. F. A. Mechanism of Polymer Collapse in Miscible Good Solvents. J. Phys. Chem. B 2015, 119, 15780-15788.

(71) Budkov, Y. A.; Kolesnikov, A. L.; Georgi, N.; Kiselev, M. G. A flexible polymer chain in a critical solvent: Coil or globule? Europhys. Lett. 2015, 109, 36005.

(72) Dignon, G. L.; Zheng, W.; Best, R. B.; Kim, Y. C.; Mittal, J. Relation between singlemolecule properties and phase behavior of intrinsically disordered proteins. Proc. Natl. Acad. Sci. U.S.A. 2018, 115, 9929-9934.

(73) Bharadwaj, S.; van der Vegt, N. F. A. Does Preferential Adsorption Drive Cononsolvency? Macromolecules 2019, 52, 4131-4138.

(74) Duboué-Dijon, E.; Fogarty, A. C.; Hynes, J. T.; Laage, D. Dynamical Disorder in the DNA Hydration Shell. J. Am. Chem. Soc. 2016, 138, 7610-7620.

(75) Ganguly, P.; van der Vegt, N. F. A.; Shea, J.-E. Hydrophobic Association in Mixed Urea-TMAO Solutions. J. Phys. Chem. Lett. 2016, 7, 3052-3059.

(76) Nayar, D.; van der Vegt, N. F. A. The Hydrophobic Effect and the Role of Cosolvents. J. Phys. Chem. B 2017, 121, 9986-9998.

(77) Remsing, R. C.; Xi, E.; Patel, A. J. Protein Hydration Thermodynamics: The Influence of Flexibility and Salt on Hydrophobin II Hydration. J. Phys. Chem. B 2018, 122, 3635-3646. 
(78) Zhang, Y.; Cremer, P. S. Chemistry of Hofmeister Anions and Osmolytes. Annu. Rev. Phys. Chem. 2010, 61, 63-83.

(79) Okur, H. I.; Hladílková, J.; Rembert, K. B.; Cho, Y.; Heyda, J.; Dzubiella, J.; Cremer, P. S.; Jungwirth, P. Beyond the Hofmeister series: ion-specific effects on proteins and their biological functions. J. Phys. Chem. B 2017, 121, 1997-2014.

(80) Mukherji, D.; Watson, M. D.; Morsbach, S.; Schmutz, M.; Wagner, M.; Marques, C. M.; Kremer, K. Soft and Smart: Co-nonsolvency-Based Design of Multiresponsive Copolymers. Macromolecules 2019, 52, 3471-3478. 


\title{
Supplementary Material
}

\section{Characterizing the Interplay between Polymer Solvation and Conformation}

\author{
Debdas Dhabal, Zhitong Jiang and Amish J. Patel* \\ Department of Chemical and Biomolecular Engineering, University of Pennsylvania, \\ Philadelphia, Pennsylvania 19104, United States
}

Three supplementary figures, which support the results presented in the main text are included.

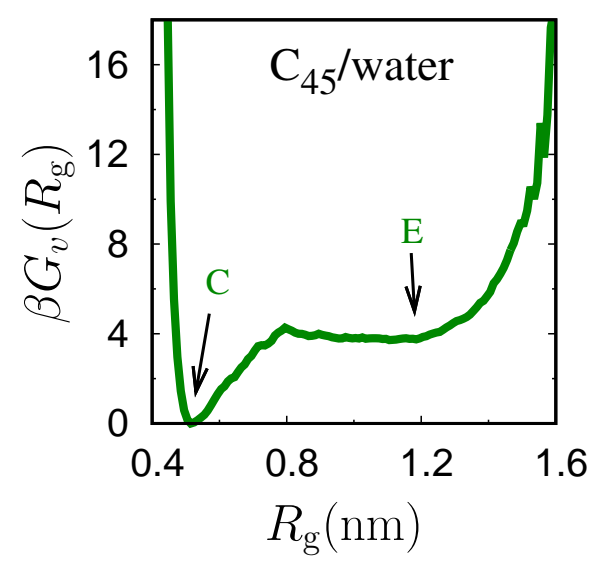

Figure S1 For the $\mathrm{C}_{45}$ polymer in water, the conformational free energy landscape, $G_{v}\left(R_{\mathrm{g}}\right)$, obtained by integrating $\exp \left[-\beta G_{v}\left(\tilde{N}, R_{\mathrm{g}}\right)\right]$ over the solvent coordinate, $\tilde{N}$, is shown as a function of the polymer radius of gyration, $R_{\mathrm{g}}$. The basins corresponding to collapsed (C) and extended (E) states are denoted by arrows. 

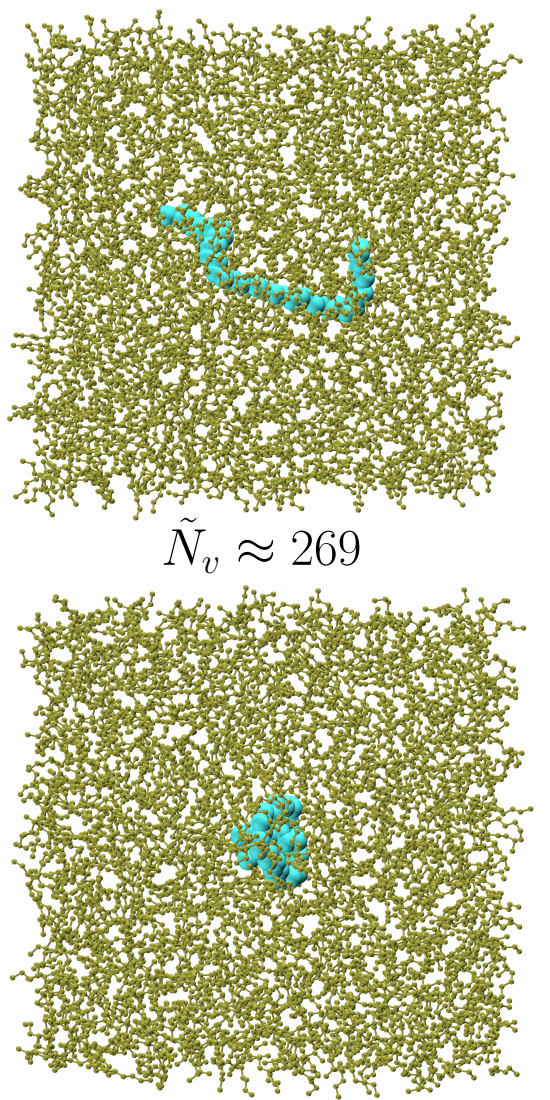

$$
\tilde{N}_{v} \approx 142
$$

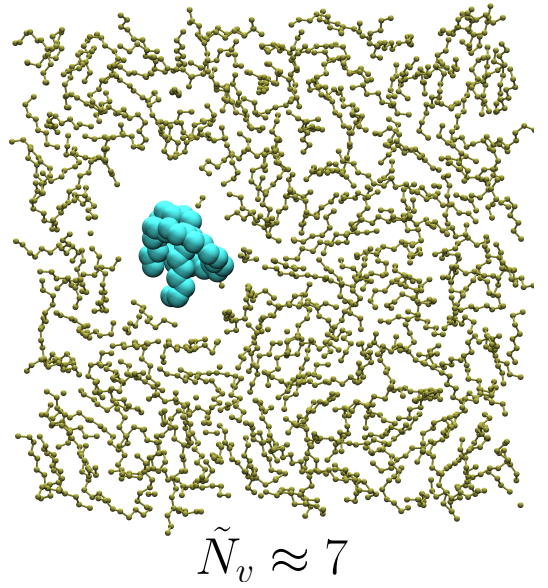

Figure S2 Simulation snapshots of the $C_{45}$ polymer (beads shown in cyan using spacefill representation) solvated in octane (gold) are shown for three select $\tilde{N}_{v}$-values. As shown in the topmost snapshot, the polymer adopts an extended configuration for $\left.\tilde{N}_{v}\right\rangle\left\langle\tilde{N}_{v}\right\rangle_{\phi_{1}^{*}}$. The middle snapshot highlights that the polymer is in a collapsed, but solvated configuration for $\left\langle\tilde{N}_{v}\right\rangle_{\phi_{2}^{*}}<\tilde{N}_{v}<\left\langle\tilde{N}_{v}\right\rangle_{\phi_{1}^{*}}$. Finally, the bottom snapshot indicates that the collapsed polymer is dewetted for $\tilde{N}_{v}<\left\langle\tilde{N}_{v}\right\rangle_{\phi_{2}^{*}}$; solvent beads in the background are hidden to clearly illustrate polymer dewetting. 


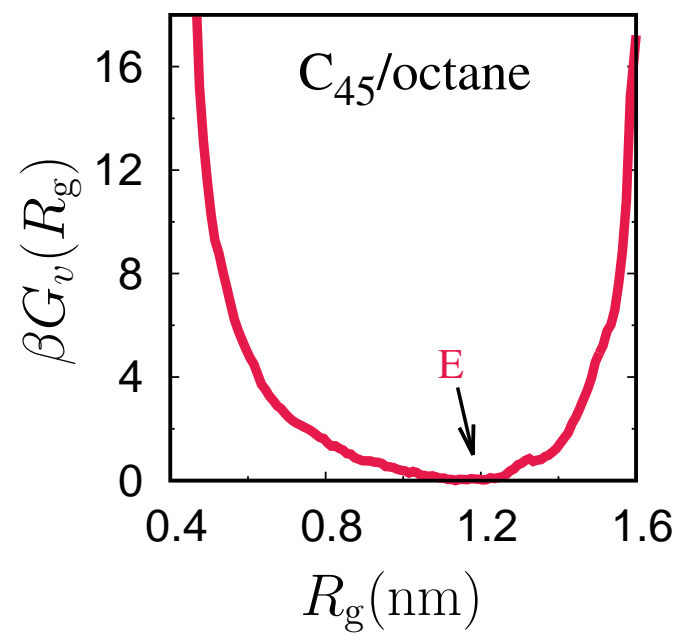

Figure S3 For the $\mathrm{C}_{45}$ polymer in octane, the conformational free energy landscape, $G_{v}\left(R_{\mathrm{g}}\right)$, obtained by integrating $\exp \left[-\beta G_{v}\left(\tilde{N}, R_{\mathrm{g}}\right)\right]$ over the solvent coordinate, $\tilde{N}$, is shown as a function of the polymer radius of gyration, $R_{\mathrm{g}}$. The basins corresponding to the extended $(\mathrm{E})$ state is denoted by an arrow; no distinct basin is observed for the collapsed state. 
Graphical TOC Entry

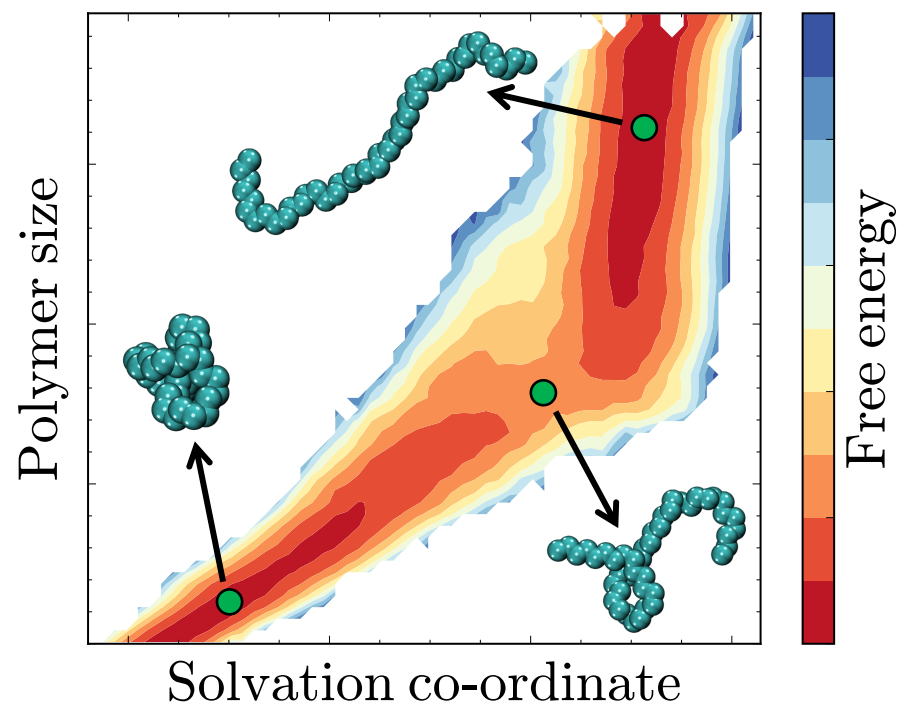

